\section{AB0939 POLYMYALGIA RHEUMATICA FOLLOWING INFECTIVE TRIGGERS OR VACCINATIONS: A DIFFERENT SUBSET OF DISEASE?}

E. Conticini ${ }^{1}$, P. Falsetti ${ }^{1}$, C. Acciai ${ }^{2}$, C. Baldi ${ }^{1}$, M. Bardelli ${ }^{1}$, S. Gentileschi ${ }^{1}$, L. Cantarini ${ }^{1}$, B. Frediani ${ }^{1}{ }^{1}$ University of Siena, Rheumatology Unit, Siena, Italy;

${ }^{2}$ San Donato Hospital, Rehabilitation Unit, Arezzo, Italy

Background: Polymyalgia rheumatica (PMR) is the most common inflammatory disorder of elderly; an association with environmental triggers and deregulated immune response has been described.

Objectives: The aim of this study was to investigate the presence of environmental triggers before the onset of PMR and its association to a particular subset of disease.

Methods: The database of 58 consecutive PMR patients recruited from a single rheumatology secondary care setting was retrospectively analyzed to investigate the frequency of environmental triggers and correlations with clinical characteristics. Patients underwent multidistrict ultrasound examination of both proximal and distal sites. Laboratory tests were repeated after one month from first visit, when steroids were started, and about every three months during follow-up (for at least 24 months)

Results: Fifteen PMR patients (26\%) described a connection with environmental agents: six PMR patients reported a vaccination, 3 an upper respiratory tract infection and 1 pneumonia before the onset of disease. Five patients reported seasonal influenza as trigger of PMR. The model of multivariate linear regression which better predicted a shorter time to normalize inflammatory reactants ( $R$ squared $27,46 \%, p=0,0042$ ) comprised the presence of an environmental trigger and a higher CRP. A linear regression analysis confirmed an inverse correlation between CRP at onset ant time to normalize inflammatory reactant $(r=-0,3031, p=0,0208)$. A significant correlation was demonstrated between presence of environmental trigger and shorter time to normalize inflammation $(r=-0,5215, p<0,0001)$, lesser frequency of gleno-humeral synovitis on US $(r=-$ $0,3774, p=0,0038$ )

Conclusion: Our work describes a correlation between environmental triggers in PMR and higher CRP at diagnosis and faster response to therapy. We may suppose that these patients belong to a more specific subtype of PMR, in whom external stimuli, such as vaccinations or infections, may lead to a deregulated response within the context of an impaired immune and endocrine system. We recommend a systematic research of previous infections or vaccination in recent onset PMR

Disclosure of Interests: None declared

DOI: 10.1136/annrheumdis-2020-eular.956

\section{$\mathrm{AB} 0940$ \\ BIOMARKERS IN THE DIAGNOSIS OF ACUTE HOT JOINTS: AN EVALUATION OF RESEARCH INTEREST 1960-2018}

M. Dey ${ }^{1,2}$, M. Al-Attar ${ }^{3}$, L. Peruffo ${ }^{4}$, I. Wilson ${ }^{1,2}$, S. S. Zhao ${ }^{1,2}$, S. Duffield ${ }^{1}$, N. Goodson ${ }^{1,2} .{ }^{1}$ Institute of Ageing and Chronic Disease, University of Liverpool, Musculoskeletal Biology I, Liverpool, United Kingdom; ${ }^{2}$ Aintree University Hospital, Liverpool University Hospitals NHS Foundation Trust, Academic Rheumatology, Liverpool, United Kingdom; ${ }^{3}$ Manchester Royal Infirmary, Manchester University NHS Foundation Trust, Manchester, United Kingdom; ${ }^{4}$ Federal University of Parana, Curitiba, Brazil

Background: The acute hot joint presentation is a common clinical emergency, often the result of crystal arthritis or trauma. However, all diagnoses can mimic septic arthritis, which should be excluded promptly due to the potential for rapid joint destruction and significant morbidity. The gold-standard test for septic arthritis is synovial fluid culture, which can take several days to perform. Meanwhile, patients are often admitted and given antimicrobials. Other specialties have made use of rapid biomarkers to exclude infection, for example, exclusion of empyema using pleural fluid $\mathrm{pH}$ and glucose [1]. Such biomarkers could reduce the need for lengthy hospital admissions and inappropriate antibiotic use in the acute hot joint presentation.

Objectives:

1.Evaluate research interest over time, on the use of diagnostic biomarkers in the acute hot joint presentation.

2.Compare research interest in the use of diagnostic biomarkers in acute hot native versus acute hot prosthetic joints.

Methods: We performed a review of the number of publications reporting the use and diagnostic accuracy of biomarkers to exclude infection in the acute hot joint presentations. The database, Scopus, was searched for English-language studies (1946-2018) using search terms relating to septic arthritis, crystal arthritis, and diagnostic markers derived from synovial fluid/aspirate. The number of papers published per year on prosthetic joints only was also calculated. Therefore, the following were recorded for each year 1946-2018: total number of studies; prosthetic joints only; native joints only. Values were plotted, with polynomial trend-lines and $\mathrm{R}^{2}$ calculated.

Results: Our search yielded 2279 relevant studies in total (561 on prosthetic joints), published 1946-2018. Only 1 study was identified for the year 1946; the next recorded publication was in 1960 . Therefore, this single study was excluded as an outlier. Results are presented in Figure 1. The number of studies on diagnostic biomarkers for acute hot joints continued to increase after 1960. From 2016, the number of studies conducted in prosthetic joints outnumbered those done in native joints. Polynomial trend-lines applied to the results showed studies on native acute hot joints are predicted to decline, while those in prosthetic joints will continue to increase.

Conclusion: Reasons for an increasing number of studies on prosthetic compared to native acute hot joints include a narrower differential diagnosis in prosthetic joints, i.e. septic vs aseptic. In contrast, native acute hot joints may be the result of various causes including crystal arthritis, inflammatory arthritis, and trauma. Having a narrower differential diagnosis may facilitate diagnostic research in prosthetic joint presentations. Furthermore, incidence of prosthetic joint infection is also greater than that of native joint infection [2]. Nonetheless, the incidence of native joint infection is increasing [3]. This, and the lack of methods by which to rapidly distinguish native joint septic arthritis from non-infective causes, indicates that more research is required in this area.

References:

[1] Heffner JE et al. Pleural fluid chemical analysis in parapneumonic effusions. A meta-analysis. Am J Respir Crit Care Med. 1995 Jun;151(6):1700-8.

[2] Roerdink RL et al. The difference between native septic arthritis and prosthetic joint infections: A review of literature. J Orthop Surg (Hong Kong).

[3] Rutherford Al et al. A population study of the reported incidence of native joint septic arthritis in the United Kingdom between 1998 and 2013. Rheumatol (United Kingdom). 2016;55(12):2176-80.

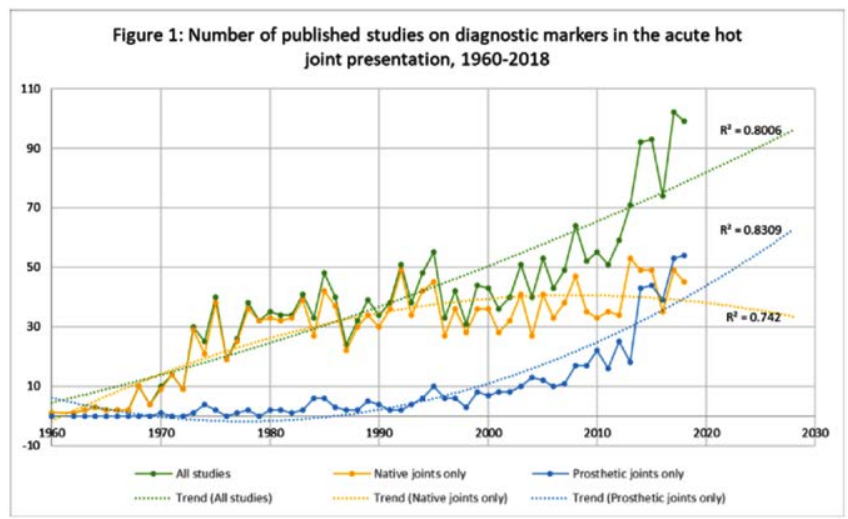

Disclosure of Interests: None declared

DOI: 10.1136/annrheumdis-2020-eular.628

\section{AB0941 \\ NEURALGIC AMYOTROPHY AND HEPATITIS INFECTION: REPORT OF 6 CASES AND REVIEW OF THE LITERATURE}

R. Garofoli', P. Seror ${ }^{2}$, J. Zauderer ${ }^{1}$, C. Nguyen ${ }^{1}$, F. Rannou ${ }^{1}$, J. L. Drapé ${ }^{1}$, A. Roren ${ }^{1}$, M. M. Lefevre Colau ${ }^{1} .{ }^{1}$ Hospital Cochin, Paris, France; ${ }^{2}$ Cabinet Electromyographie - Paul Seror, Paris, France

Background: Neuralgic amyotrophy (NA) or Parsonage and Turner syndrome is triggered at least in $25 \%$ by a viral infection: parvovirus B19, CMV, HSV, etc.. Recently, few cases of Hepatitis E Virus (HEV) related NA were reported. This particular association remains little known and is overlooked by most physicians. Besides, clinical, electrodiagnostic (EDX) and MRI characteristics, as well as evolution of HEV-related NA have not been fully described yet.

Objectives: To describe 6 cases of HEV-related NA and to perform a review of the literature.

Methods: We describe longitudinally clinical examination, electrodiagnostic (EDX), biological and MRI results of 6 cases of HEV-associated NA, diagnosed in our center.

Results: The 6 cases were aged between 33 and 57 years old (mean 44.5), sex ratio was $5 \mathrm{M} / 1 \mathrm{~F}$. All patients had positive IgM anti-HEV (serology) and a cervica MRI that could not explain clinical presentation. Overall, the 6 patients totalize 26 mononeuropathies (range 1 to 8 per patient), 5/6 patients had a severe presentation of NA, with bilateral and asymmetric symptoms (3 cases). HEV-related NA involved classical nerves such as supra-scapular (6 cases, twice bilaterally) and long thoracic nerves (5 cases), some less classical nerves like anterior 